\title{
INDISSOCIABILIDADE ENTRE ENSINO, PESQUISA E EXTENSÃO: RELATO DE EXPERIÊNCIAS DO CURSO DE SISTEMAS DE INFORMAÇÃO DA UFSM/FREDERICO WESTPHALEN
}

\author{
THE STRAIGHT CONNECTION AMONG TEACHING, RESEARCH AND \\ EXTENSION: AN EXPERIENCE REPORT FROM THE BACHELOR OF \\ COMPUTER INFORMATION SYSTEMS OF UFSM/FREDERICO WESTPHALEN
}

Sidnei Renato Silveira ${ }^{1}$, Cristiano Bertolini ${ }^{1}$, Fábio José Parreira ${ }^{1}$

\begin{abstract}
RESUMO
Este artigo apresenta uma das formas de viabilizar a indissociabilidade entre ensino, pesquisa e extensão - um dos fundamentos da Educação Superior. No Curso de Sistemas de Informação da UFSM (Universidade Federal de Santa Maria) - Campus Frederico Westphalen - RS, o ensino, a pesquisa e a extensão são promovidos de diferentes formas e, em especial, por meio da realização do TGSI (Trabalho de Graduação em Sistemas de Informação). O TGSI é desenvolvido em duas disciplinas obrigatórias, no último ano do curso, sob orientação de um ou mais docentes, visando ao desenvolvimento de um projeto. Este projeto pode estar ligado ao ensino, à pesquisa e/ou à extensão, independentemente ou simultaneamente, de acordo com o tipo de projeto desenvolvido. Neste contexto, este artigo apresenta um relato de experiência desenvolvida nas disciplinas de TGSI do referido curso, destacando a indissociabilidade entre ensino, pesquisa e extensão, por meio de projetos, tais como protótipos de software, desenvolvidos com inserção local e regional.

Palavras-chave: Ensino; Pesquisa; Extensão.
\end{abstract}

\begin{abstract}
This paper presents one way to enable the straight connection among teaching, research and extension - one of the foundations of the higher education. In the Bachelor of Computer Information Systems of UFSM (Federal University of Santa Maria)/Frederico WestphalenRS, the teaching, research and extension are promoted in different contexts and, in particular, by the final dissertation called TGSI. The TGSI comprehends two courses in the last year of the bachelor, under supervision of one or more professors in order to conclude the dissertation. This project can be connected to the teaching, research and/or extension in an independently or simultaneously way based on the type of work developed by the student. In this context, this paper presents an experience report in the TGSI courses, emphasizing the straight connection among teaching, research and extension through students projects like software prototypes to the local software companies.
\end{abstract}

\footnotetext{
${ }^{1}$ Departamento de Tecnologia da Informação. UFSM - Universidade Federal de Santa Maria. Campus Frederico Westphalen - RS.
} 
Keywords: Teaching; Research; Extension.

\section{INTRODUÇÃo}

A organização didático-pedagógica do Curso de Bacharelado em Sistemas de Informação (SI) da Universidade Federal de Santa Maria (UFSM) - Campus Frederico Westphalen - RS, é baseada na indissociabilidade entre o ensino, a pesquisa e a extensão, como preconiza a Lei de Diretrizes e Bases da Educação Nacional (LDB 9394/96) (BRASIL, 1996). Na indissociabilidade, referida como princípio pedagógico de organização do curso, em termos de ensino com pesquisa, como ação educativa, a mesma acontece quando os alunos adquirem autonomia intelectual para que possam aprender sempre, além das atividades ligadas à pesquisa institucional docente, preocupada com a construção do conhecimento na área de Informática. Por outro lado, em termos de ensino com extensão, há a qualificação das atividades complementares, pelo leque de oportunidades que asseguram a flexibilização da estrutura curricular e pela contextualização sócio-econômica, política e cultural. O ensino junto com a extensão também permite a indivisibilidade entre a teoria e a prática, na medida em que insere a prática com vistas à formação profissional desde o início do curso, articuladamente com a comunidade, não só contextualizando o ensino, mas oportunizando o desenvolvimento de sua política de responsabilidade social (UFSM, 2016).

A UFSM tem como missão promover ensino, pesquisa e extensão, formando lideranças capazes de desenvolver a sociedade, ou seja, de gerar e difundir conhecimento e não apenas reproduzi-lo, o que exige capacitação e responsabilidade por parte dos segmentos docente, discente e técnico-administrativo, em colaborar para a manutenção de uma Universidade pública, gratuita e de qualidade (UFSM, 2000).

A elaboração de diferentes projetos ao longo do curso, tais como projetos de pesquisa e de extensão (de iniciativa docente) e projetos desenvolvidos em disciplinas (tais como as atividades desenvolvidas em disciplinas integradoras - Projeto de Software I e II), viabiliza a construção da indissociabilidade entre ensino, pesquisa e extensão, já que os conteúdos estudados nas disciplinas (ensino) são aplicados nos diferentes projetos desenvolvidos (pesquisa e extensão) e estes conteúdos realimentam as atividades de ensino que, continuamente, refletem-se nos projetos de pesquisa e de extensão, em um ciclo contínuo.

Entre os projetos desenvolvidos durante o Curso de SI, destaca-se o TGSI (Trabalho de Graduação em Sistemas de Informação), que é o trabalho de conclusão do curso, 
desenvolvido pelos alunos, sob orientação dos docentes, no último ano do currículo. Tendo-se em vista a indissociabilidade entre ensino, pesquisa e extensão, bem como o cumprimento dos objetivos do curso e o alinhamento ao perfil de egresso desejado, os TGSIs, constantemente, refletem, compreendem e articulam-se em projetos ligados ao ensino, à pesquisa e/ou à extensão, muitas vezes perpassando mais de um destes pilares simultaneamente.

Deste modo, observando que a legislação brasileira, em especial a LDB 9394/96 (BRASIL, 1996), bem como a propostas de Diretrizes Curriculares para os cursos de área de Computação (MEC, 2012), priorizam não só os conteúdos universais, mas também o desenvolvimento de competências e habilidades na busca do aperfeiçoamento da formação cultural, técnica e científica do cidadão, o Projeto Pedagógico do Curso de Graduação em Sistemas de Informação busca, preponderantemente, a garantia da flexibilidade curricular, da formação integral do cidadão, da interdisciplinaridade, da articulação entre teoria e prática, e da indissociabilidade entre ensino, pesquisa e extensão (UFSM, 2016).

Neste contexto, este artigo apresenta um relato da experiência realizada nas disciplinas de TGSI do Curso de SI da UFSM/FW, destacando de que forma a indissociabilidade entre ensino, pesquisa e extensão acontece na prática.

Cabe destacar que o Curso de SI da UFSM/FW é, atualmente, o único curso noturno do campus de Frederico Westphalen. Sendo assim, a grande maioria dos acadêmicos atua profissionalmente durate o dia e, muitos acadêmicos, atuam em empresas da área de TI (Tecnologia da Informação) na região. Além disso, faz-se importante destacar a percepção de pesquisa e de extensão de acordo com o PPC (Projeto Pedagógico de Curso) e com o perfil de egresso pretendido. Neste sentido, entende-se as atividades de investigação como pesquisas aplicadas, voltadas, na sua grande maioria, para a solução de problemas que envolvem a TI (RIBEIRO; ZABADAL, 2010).

Com relação à extensão, o PPC do Curso de SI destaca que, dentre os pilares das atividades extensionistas, destaca-se a articulação entre teoria e prática, estimulando a formação profissional dos acadêmicos de forma articulada com a comunidade, o que pode ser realizado por meio das atividades desenvolvidas em parceria com as empresas da região. Neste artigo entende-se que a pesquisa aplicada, por meio da solução de problemas do mundo do trabalho, no contexto local e regional, alinha-se à extensão tecnológica, caracterizando projetos que envolvem ensino, pesquisa e extensão. 
Para tanto, o artigo está organizado como segue: na seção 2 apresentam-se algumas informações referentes ao PPC de SI da UFSM/FW, destacando a indissociabilidade entre ensino, pesquisa e extensão como princípios basilares. Na seção 3 destaca-se, de forma sucinta, como são conduzidas as disciplinas de TGSI. A seção 4 apresenta alguns TGSIs desenvolvidos, destacando-se seus enfoques de pesquisa e/ou de extensão. Encerrando o artigo, apresentam-se as considerações finais e as referências empregadas.

\section{INDISSOCIABILIDADE ENTRE ENSINO, PESQUISA E EXTENSÃO NO PPC DO CURSO DE SI/UFSM/FW}

Tendo em mente a indissociabilidade entre ensino, pesquisa e extensão, o objetivo geral do Curso de SI da UFSM/FW é o de formar profissionais de nível superior com domínio e capacidade para trabalhar na área da Computação, desenvolvendo projetos de software e serviços de computação e informação, atentos ao caráter ecológico, social, ético e responsável no exercício de suas atividades na sociedade (UFSM, 2016).

Entre os objetivos do curso, podem-se destacar a pesquisa e a extensão, quando se estabelece que os alunos devem estar aptos a intervir na realidade, de forma empreendedora e criativa. Acredita-se que as atividades de pesquisa e de extensão potencializem a criatividade e estimulem o empreendedorismo por parte dos docentes e discentes do curso.

Com relação ao impacto de suas atividades na sociedade, que podem ser por meio de projetos de extensão ou de pesquisa aplicada, os objetivos do curso, estabelecidos no PPC, destacam que os alunos devem desenvolver conhecimentos e habilidades humanas, sociais e gerenciais que possibilitem compreender o impacto da tecnologia no arranjo social, de modo a assumir uma atitude consciente e pró-ativa, utilizando a TI como ferramenta para a otimização de processos nas organizações e transformação social.

O Curso de SI da UFSM/FW, na busca de uma identidade clara, considera estratégias pedagógicas que enfatizem a construção - produção do conhecimento, ao invés da (simples) transmissão e aquisição de informações. Neste sentido, o curso, além de metodologias demonstrativas (ex: aulas expositivas) procura diversificações didático- pedagógicas que privilegiem a pesquisa e a extensão como instrumentos de aprendizagem, estimulando a atitude científica e profissional. Para tanto, o curso promove a inserção dos alunos e professores em grupos de ensino, pesquisa e extensão que tragam benefícios para a qualidade e aperfeiçoamento do ensino, para a gestão universitária e para a sociedade. Pimenta e 
Anastasiou (2008, p. 91) destacam que: "Em todos os níveis dos cursos universitários deve prevalecer sempre a interação e a pesquisa, e não a mera transmissão de conhecimentos".

O ensino com pesquisa, segundo Anastasiou e Alves (2003, p. 98), "oferece condições para que os estudantes adquiram maior autonomia, assumam responsabilidades, desenvolvam disciplina [...]", entre outras habilidades importantes para a formação profissional dos acadêmicos.

De acordo com o perfil profissional traçado, o egresso do Curso de SI da UFSM/FW deve estar apto a desenvolver as seguintes funções no mercado de trabalho:

- empreendedor - descobrimento e empreendimento de novas oportunidades para aplicações usando sistemas computacionais e avaliando a conveniência de se investir no desenvolvimento da aplicação/serviço;

- consultor - consultoria e assessoria a empresas de diversas áreas no que tange ao uso adequado de Sistemas de Informação;

- coordenador de equipe - coordenação de equipes envolvidas em projetos ligados à TI;

- membro de equipe - participação de forma colaborativa e integrada de equipes que desenvolvem projetos na área de TI;

- pesquisador - participação em projetos de pesquisa científica e tecnológica.

A consolidação do PPC do Curso de SI da UFSM/FW exige o esforço de todos os envolvidos no processo. Neste contexto, o corpo docente deve estar consciente do seu papel, enquanto sujeito envolvido e responsável pela efetivação do Projeto Pedagógico, e deve assumir comportamentos e atitudes no desempenho de suas funções, visando atingir os objetivos do Curso.

Neste sentido, partindo-se do pressuposto da indissociabilidade entre o ensino, pesquisa e extensão, com relação à metodologia e atitudes do corpo docente, cada docente deve atuar promovendo a:

- interação entre os objetivos da UFSM e do Curso, por meio de ações devidamente articuladas e cooperativas, visando a efetivação do PPC;

- capacitação e atualização científica e didático-pedagógica;

- inserção do curso na comunidade científica profissional, por meio da participação em comissões científicas, movimentos associativos, grupos de pesquisa, eventos científicos e profissionais; 
- integração com o corpo discente por meio das práticas pedagógicas, de orientação acadêmica, da iniciação científica, de estágios e monitorias;

- divulgação e socialização do saber através de produções científicas, técnicas e culturais;

- inserção do curso no contexto institucional, participando da gestão acadêmica e administrativa;

- inserção do curso no contexto social por meio de práticas extensionistas, ações comunitárias e integração com a comunidade e grupos de pesquisa;

- valorização e ênfase da dimensão multidisciplinar e do trabalho multiprofissional, bem como da integração das disciplinas do currículo do curso;

- valorização e utilização dos resultados do processo de avaliação institucional como meio de promover a melhoria do ensino no âmbito do Curso de SI.

As atividades desenvolvidas em sala de aula devem aliar o tripé defendido pelo Projeto Pedagógico Institucional (PPI), que defende a indissociabilidade entre a pesquisa, o ensino e a extensão, permitindo que os alunos tenham a oportunidade de participar de atividades que requeiram atitudes mais protagonistas. Essas atividades de pesquisa e extensão são importantes porque introduzem os alunos nas problemáticas relacionadas às áreas de conhecimento do curso, assim como os põem em contato com a realidade local, regional, nacional e internacional (UFSM, 2000).

\section{TGSI NO CURSO DE SI/UFSM/FW}

Ao final do Curso de SI, o aluno deverá cursar as disciplinas de Trabalho de Graduação (TGSI) I e II, totalizando 240 horas, de acordo com as Normas para Realização do Trabalho de Graduação (UFSM, 2013).

Estas disciplinas podem ser consideradas uma das partes mais importantes do curso, pois se trata de uma oportunidade que o aluno tem de demonstrar independência e originalidade. Nela o aluno deverá ser capaz de planejar, organizar e realizar um projeto, bem como desenvolvê-lo utilizando os métodos e técnicas estudadas durante o curso. Do ponto de vista do aluno, qualquer que seja seu nível de desempenho acadêmico, por meio do TGSI ele tem a chance de demonstrar todo o seu potencial realizador, inovador e criativo, tanto quanto a sua capacidade de "vender seu produto", seja sob forma escrita (por meio de documento que 
poderá inclusive servir a outros), seja sob forma de apresentação oral. O resultado final deverá trazer um sentimento de "ter realizado algo, com começo, meio e fim".

Tecnicamente, um projeto de TGSI envolve a definição, modelagem e implementaçãopiloto de uma aplicação, metodologia, produto ou serviço de software com características inovadoras. Os projetos inovadores, invariavelmente, avançam fronteiras, tais como o desenvolvimento de uma aplicação que ainda não existe, ou melhora significativa de uma aplicação ou método já existente, seja através de sua funcionalidade ou desempenho, dentre outras propriedades.

A estrutura curricular (currículo 2016) do Curso de SI da UFSM/FW prevê a sua realização no $8^{\circ}$ e $9^{\circ}$ semestres do Curso, nas disciplinas de Trabalho de Graduação em Sistemas de Informação I e II, respectivamente. Cada disciplina possui 120 horas, divididas em 8 créditos, sendo 2 créditos teóricos e 6 créditos práticos (UFSM, 2016).

Durante o TGSI o aluno deverá desenvolver estudos ou aplicações sobre temas relacionados com o Curso. Este trabalho será regido por regulamento próprio e compreenderá a realização de um trabalho de caráter teórico-prático, condizente com a formação oferecida pelo Curso, que será supervisionado por um professor orientador e implicará na elaboração de um artigo científico e defesa perante uma banca examinadora (UFSM, 2013).

O TGSI objetiva proporcionar ao estudante a oportunidade de consolidar os conhecimentos adquiridos no curso através da reflexão de como o aprendizado teórico (conhecimento sistematizado) é aplicado à identificação de problemas organizacionais concretos, mediante atividade em organizações reais, tendo como objetivo principal o aprimoramento e a integração dos conhecimentos e dos conteúdos do curso, tendo em vista a atuação do futuro profissional.

O principal objetivo do TGSI é o de propiciar ao aluno a oportunidade de consolidar os conhecimentos construídos e compartilhados durante seu envolvvimento no curso de Sistemas de Informação, por meio da reflexão de como o aprendizado teórico é aplicado à solução dos problemas computacionais. Esta atividade envolve, preponderantemente, a investigação do conhecimento (PIMENTA; ANASTASIOU, 2008).

No final do semestre, os resultados obtidos são apresentados perante uma banca examinadora. Além desta apresentação, o TGSI será avaliado mediante a entrega de um documento no estilo de um artigo científico, de acordo com as normas da SBC - Sociedade Brasileira de Computação, da ACM - Association for Computing Machinery, IEEE - Institute 
of Electrical and Electronics Engineers ou LNCS - Lecture Notes in Computer Science. Sugere-se, sempre que possível, que o artigo seja submetido a eventos e/ou periódicos científicos. A aprovação do artigo em eventos e/ou periódicos poderá ser utilizada como um diferencial na atribuição do conceito do trabalho.

Entre as alternativas que permite a manutenção da indissociabilidade entre ensino, pesquisa e extensão, destacam-se: 1) a vinculação de projetos de pesquisa e/ou de extensão a disciplinas (tais como as disciplinas de TGSI I e II) e 2) o incentivo ao desenvolvimento de projetos de investigação local e regional. Estas alternativas estão elencadas no Projeto Pedagógico Institucional da UFSM (UFSM, 2000). No contexto do segundo item destacam-se a aplicação dos resultados dos TGSIs em empresas e escolas da região, abordados na próxima seção.

\section{PROJETOS DESENVOLVIDOS: A INDISSOCIABILIDADE ENTRE ENSINO, PESQUISA E EXTENSÃO NA PRÁTICA}

O Curso de SI da UFSM/FW já formou 18 Bacharéis em Sistemas de Informação, em 3 diferentes turmas, sendo que 22 TGSIs já foram concluídos (4 alunos que concluíram o TGSI ainda não colaram grau). Os trabalhos realizados perpassam os três pilares da Educação Superior - ensino, pesquisa e extensão, mostrando, de forma prática, como estas áreas são indissociáveis.

O ensino fica evidente durante a aplicação das técnicas, tecnologias e metodologias que permitem o desenvolvimento dos mais diferentes TGSIs e envolvem, por exemplo, o estudo e aprofundamento de diversas áreas e temáticas abordadas durante o curso, tais como Linguagens de Programação, Bancos de Dados, Engenharia de Software, Interação HumanoComputador, Inteligência Artificial, Qualidade de Software, Redes de Computadores e Gestão da Tecnologia da Informação, entre outras.

Com relação ao pilar da extensão, destaca-se que muitos TGSIs são destinados a solucionar problemas das empresas da região. Isto se deve ao fato de que muitos acadêmicos atuam profissionalmente na área de TI, mesmo antes de concluírem o curso, e desenvolvem seus TGSIs nas empresas onde atuam. Isto permite a aplicação dos conteúdos estudados no curso (ensino) na prática, solucionando problemas reais do mundo do trabalho. Esta inserção nas empresas amplia o conhecimento gerado na Universidade para a comunidade, o extramuros, um dos pilares da extensão. Esta inserção não só permite o compartilhamento dos 
conhecimentos gerados na Universidade com a comunidade mas, também, permite que o conhecimento seja realimentado, de acordo com a realidade e as necessidades do mundo do trabalho. Pimenta e Anastasiou (2008) destacam que, os alunos apontam o problema da distância dos cursos universitários com a realidade. A realização de atividades, envolvendo as empresas da região, permitem reduzir esta distância, aproximando a academia do mundo do trabalho.

A solução dos problemas reais da comunidade onde a universidade está inserida, por meio de atividades de pesquisa e/ou de extensão, reforça, também, o compromisso social da instituição. O compromisso social (ou responsabilidade social) da universidade, segundo Goergen (2006), deve envolver a criação de condições para a produção de conhecimentos e saberes, visando formar bons profissionais nas suas áreas de atuação.

Para desenvolver as soluções necessárias para as empresas os alunos precisam, também, estudar novas ferramentas, técnicas, tecnologias, etc., fortalecendo as atividades de pesquisa. A pesquisa, ou investigação, busca a geração do conhecimento. Nos casos em que os TGSIs envolvem a solução de problemas do mundo do trabalho, tem-se a pesquisa aplicada (RIBEIRO; ZABADAL, 2010). Estes TGSIs apresentam, na prática, o exemplo mais concreto da indissociabilidade entre ensino, pesquisa e extensão. $\mathrm{O}$ ensino, por meio dos conteúdos que os alunos estudam durante o curso e aplicam nos TGSIs, a extensão, com a inserção da universidade na comunidade (extramuros) - em especial a ideia da extensão tecnológica - e a pesquisa, por meio da pesquisa aplicada, visando solucionar problemas do mundo do trabalho.

Entre as empresas e instituições que tiveram problemas solucionados por meio do desenvolvimento de TGSIs, destacam-se:

- $\quad$ Alliance Software, localizada no município de Palmeira das Missões-RS, por meio de um estudo de caso sobre a aplicação de metodologias ágeis no desenvolvimento de software (CONCEIÇÃO; SILVEIRA, 2015);

- Arbaza Alimentos, de Frederico Westphalen-RS: desenvolvimento de um Sistema Inteligente de Apoio à Decisão (SIAD) como Ferramenta de Business Intelligence (FRIZON et al., 2015);

- $\quad$ Digifred Sistemas de Gestão Pública - Frederico Westphalen-RS, com o TGSI envolvendo um estudo de caso sobre a implementação do MPS.BR na empresa (CERATTI et al., 2014); 
- $\quad$ Exatus Soluções Estratégicas, de Ijuí-RS: implantação de um sistema de Help Desk (LINKE; SILVEIRA, 2015);

- $\quad$ Pizzaria Fratello, de Rodeio Bonito-RS: implementação de um sistema de pedidos e de recomendação de pizzas, acessível por meio de browsers web e dispositivos móveis (GARBIN; SILVEIRA, 2014);

- $\quad$ Prefeitura Municipal de Frederico Westphalen-RS: desenvolvimento de um Sistema Especialista para Licenciamento Ambiental (ALMEIDA et al., 2015);

- $\quad$ Prefeitura Municipal de Palmeira das Missões-RS: implantação de um ambiente de segurança na rede de computadores (MOLINA et al., 2015);

- $\quad$ Supermercados Barril: implementação de um site de comércio eletrônico (MOERSCHBÄCHER; SILVEIRA, 2015).

Outras empresas também foram atendidas mas, por questões de sigilo estabelecidas pelas mesmas, seus nomes foram omitidos nos TGSIs:

- $\quad$ Proposta de um processo de teste de software (PRETTZ; BERTOLINI, 2015);

- Desenvolvimento de uma ferramenta para apoio ao suporte técnico, empregando Raciocínio Baseado em Casos, para um provedor de Internet da região (FERRARI et al., 2015);

- $\quad$ Desenvolvimento de um modelo para aplicação de RFID na rastreabilidade dos produtos de um frigorífico (PREDIGER et al., 2016).

Ainda considerando o pilar da pesquisa, alguns TGSIs também enfocam a pesquisa aplicada, apesar de não serem desenvolvidos, especificamente, para atender a uma demanda do mundo do trabalho. Destacam-se, neste contexto, os trabalhos de:

- Zacarias et al. (2015), “Formal Analysis of a Bio-Inspired Wireless Sensor Network Routing Algorithm";

- $\quad$ Prado e Silveira (2015): desenvolvimento de um Framework de Recomendação para lojas virtuais;

- $\quad$ Zanardi e Silveira (2015): pesquisa, do tipo survey, que traçou o perfil dos profissionais e das empresas de TI de Frederico Westphalen-RS;

Vieira et al. (2015): desenvovlimento de um Sistema de Auxílio ao Diagnóstico de Diabetes empregando Redes Neurais (SADD). 
Com relação à extensão, alguns TGSIs são desenvolvidos a partir de projetos extensionistas dos docentes. Para o desenvolvimento destes TGSIs os acadêmicos precisam, também, aliar ensino e pesquisa. Exemplos destes TGSIs envolvem os seguintes trabalhos:

Murari e Cunha (2014), elaboraram um TGSI ligado a um projeto de extensão desenvolvido no âmbito do Hospital Divina Providência, inserido no grupo de pesquisa em Informática Aplicada à Saúde, que envolve docentes e profissionais ligados à área de Saúde (CNPq, 2016b);

- $\quad$ Ceratti e Bertolini (2015) desenvolveram um projeto de extensão relacionado à Governança de Tecnologia de Informação, usando como estudo de caso o próprio campus da universidade;

Piovesan, Bertolini e Cunha (2015) pesquisaram as normas de acessibilidade na web e propuseram uma classificação das normas atuais onde, para cada norma, são geradas regras que podem ou não serem automatizadas. Este projeto está inserido no grupo de Pesquisa CTA (Comunicação, Tecnologia e Acessibilidade), que envolve docentes e alunos dos cursos de Sistemas de Informação e de Jornalismo.

Alguns trabalhos já desenvolvidos têm seu enfoque na Informática na Educação, por meio do desenvolvimento de softwares educacionais, tais como jogos educacionais digitais. Estes jogos são voltados para crianças em idade escolar e são validados em escolas da rede pública (Municipais e Estaduais) e da rede privada da região, ampliando, novamente, a inserção da universidade e o caráter extramuros das atividades. Estes projetos têm sido desenvolvidos no âmbito do Grupo de Pesquisa IATE-UFSM (Inteligência Artificial e Tecnologia Educacional) (PARREIRA et al., 2015; CNPq, 2016a). O desenvolvimento destes jogos também une ensino e pesquisa, como acontece nos projetos desenvolvidos para as empresas locais. Entre os jogos educacionais desenvolvidos destacam-se:

- Jogo educacional digital para auxílio à alfabetização, aplicando Redes Neurais (BASSO et al., 2015);

- Jogo educacional digital para apoio ao aprendizado da Matemática (KLISZCZ et al., 2016).

O desenvolvimento dos TGSIs realimenta as atividades de extensão e de pesquisa, no momento em que os docentes e discentes descobrem novos problemas e alternativas de solução que podem ser aplicadas de acordo com o contexto regional.

Além disso, Pereira (2006, p. 164-165) coloca que 
Vivemos em uma era em que ciência, tecnologia e desenvolvimento tecnológico constituem elementos estratégicos para aumentar a competitividade de um país, no que diz respeito ao conhecimento, e melhorar a qualidade de vida de sua população. [...] Nesse contexto é que, nos países em desenvolvimento como o Brasil, sistema universitário, especialmente público, se coloca como o principal pilar de sustentação do desenvolvimento da ciência e da tecnologia da nação [...].

Neste sentido, acredita-se que o desenvolvimento dos TGSIs está contribuindo, efetivamente, para o desenvolvimento da ciência e da tecnologia, em especial na região onde o Curso de SI da UFSM/FW está inserido.

\section{CONSIDERAÇÕES FINAIS}

O desenvolvimento dos TGSIs, seguindo o preceito da indissociabilidade entre ensino, pesquisa e extensão, permite que os alunos desenvolvam uma série de habilidades, tais como 1) a reflexão crítica da realidade - quando desenvolvem trabalhos ligados a projetos extensionistas ou de solução de problemas do mundo do trabalho; 2) o estímulo à participação e protagonismo, compreendendo o aluno como sujeito ativo nos processos de ensino e de aprendizagem, possibilitando que os alunos descubram que são capazes de produzir e redescobrir conhecimentos; 3) o estímulo à leitura, reflexão e escrita, habilidades necessárias para a elaboração dos artigos científicos (CUNHA et al., 2001). Bocchese (2001) também destaca a importância da escrita como processo no Ensino Superior, permitindo a comunicação, ao grande público, dos resultados dos projetos de pesquisa e/ou de extensão realizados na universidade.

Cabe destacar que, para que o resultado dos TGSIs sejam positivos, professores e alunos precisam dedicar um tempo maior de orientação (no caso dos professores) e de estudos (no caso dos alunos). O tempo e o volume de trabalho, quando se atua visando à construção do conhecimento, são muito maiores do que o dispendido em uma aula expositiva tradicional (CUNHA et al., 2001).

Os resultados dos TGSIs têm sido publicados em eventos e diferentes periódicos científicos. Dos 22 TGSIs concluídos até o momento, 7 (sete) - 31,81\% -tiveram seus resultados publicados em periódicos científicos e outros 7 (sete) publicados em anais de eventos científicos na área de Computação, atingindo-se o percentual de 63,63\% de TGSIs publicados. Outros 7 (sete) trabalhos encontram-se em fase de avaliação em diferentes periódicos. O Quadro 1 apresenta o detalhamento dos resultados dos TGSIs publicados, por meio de artigos científicos, em periódicos e/ou anais de eventos. 
Quadro 1. Publicação dos Resultados dos TGSIs.

\begin{tabular}{|c|c|}
\hline $\begin{array}{c}\text { Publicação dos Resultados } \\
\text { dos TGSIs }\end{array}$ & $\begin{array}{c}\text { Periódico ou Evento }- \text { Ano }- \text { Quantidade } \\
\text { Periódicos Científicos }\end{array}$ \\
Revista Jr. ICCEEG $-2015-1$ artigo \\
Revista RCT $-2015-1$ artigo \\
Revista Tear IFRS $-2016-1$ artigo \\
Revista RSC $-2016-2$ artigos \\
Revista REABTIC -1 artigo \\
\hline Anais de Eventos & EATI - 2014 - 4 artigos curtos \\
& EATI - 2015 - 1 artigo completo e 1 artigo curto \\
& Computer on the Beach $-2015-1$ resumo expandido \\
\hline
\end{tabular}

Destacando-se a indissociabilidade entre ensino, pesquisa e extensão, preconizada nos documentos da UFSM, tanto no PPI (UFSM, 2000) quanto no PPC do Curso de SI da UFSM/FW (UFSM, 2016), agruparam-se os TGSIs desenvolvidos e em desenvolvimento (totalizando 44 trabalhos) em categorias, como mostra o Quadro 2.

Quadro 2. Categorização dos TGSIs.

\begin{tabular}{|c|c|}
\hline Categorias Definidas & $\begin{array}{c}\text { Quantidade de TGSIs } \\
\text { (concluídos e/ou em } \\
\text { desenvolvimento) }\end{array}$ \\
\hline docente & 3 \\
\hline TGSIs ligados a projetos de extensão de iniciativa \\
docente
\end{tabular}


Os dados apresentados no Quando 2 demonstram que a realização dos TGSIs está alinhada à vocação do curso de SI da UFSM/FW, no que tange ao desenvolvimento de projetos que estimulem o desenvolvimento científico e tecnológico regional e, no que tange à indissociabilidade entre ensino, pesquisa e extensão, destacam-se os 26 TGSIs envolvendo a pesquisa aplicada alinhada à extensão tecnológica.

Considerando o perfil do egresso, definido no PPC do Curso de SI da UFSM/FW (UFSM, 2016), a indissociabilidade entre ensino, pesquisa e extensão, colocada em prática no TGSI, permite que os alunos 1) desenvolvam atitudes empreendedoras, 2) atuem em projetos que beneficiam a comunidade local e regional, 3) vivenciem a participação em projetos de pesquisa e/ou de extensão e 4) atuem em empresas da região. A atuação em empresas da região, especialmente na área do curso - Tecnologia da Informação, é uma realidade, já que a maioria dos TGSIs (desenvolvidos e/ou em desenvolvimento), como mostra o Quadro 2, envolvem a participação de empresas. Estes dados comprovam a inserção do curso na comunidade local, bem como a vocação do Curso de Sistemas de Informação, que é a de aplicar os recursos de informática como uma atividade meio, beneficiando a sociedade por meio do desenvolvimento de soluções baseadas em TI. Outro foco é a Educação Continuada. Neste sentido, destaca-se que, dos 18 egressos do Curso de SI da UFSM/FW, 6 estão cursando mestrado em diferentes instituições, tais como UFRGS (Universidade Federal do Rio Grande do Sul), USP (Universidade de São Paulo) e UnB (Universidade de Brasília) e 4 estão cursando cursos de Pós-graduação Lato Sensu, representando 55,55\% dos alunos já formados que continuaram seus estudos formalmente.

\section{REFERÊNCIAS}

ALMEIDA, L. R.; SILVEIRA, S. R.; CUNHA, Guilherme Bernardino. Ambiental Web: Sistema Especialista para Apoio à Avaliação de Processos de Licenciamento Ambiental. Anais do VI EATI - Encontro Anual de Tecnologia da Informação, 2015. Disponível em: <http://eati.info/eati/2015/assets/anais/index.html>. Acesso em: mai. 2016.

ANAStASIOU, L. G. C.; ALVES, L. P. (Org.) Processos de Ensinagem na Universidade: pressupostos para as estratégias de trabalho em aula. Joinville, SC: UNIVILLE, 2003.

BASSO, M. et al. Desenvolvimento de um Jogo Digital para Auxílio à Alfabetização utilizando Redes Neurais. Frederico Westphalen-RS: UFSM, 2015. Disponível em: <http://w3.ufsm.br/frederico/images/DesenvolvimentodeumJogoEducacionalDigitalpa raAuxilioaAlfabetizacaoempregandoRedesNeurais.pdf>. Acesso em: jun. 2016. 
BOCCHESE, J. C. A Escrita como Processo no Ensino Superior. In: FERNANDES, C. M. B.; GRILLO, M. (Orgs.) Educação Superior: travessias e atravessamentos. Canoas: Ed. ULBRA, 2001.

BRASIL. Lei 9.394 de 20 de dezembro de 1996: estabelece as diretrizes e bases da educação nacional. Disponível em: <http://www.planalto.gov.br/ccivil_03/leis/L9394.htm>. Acesso em: jun. 2016.

CERATTI, P.; BERTOLINI, C.; SILVEIRA, S. R. Implementação do MPS.BR na empresa Digifred Sistemas para Gestão Pública: um estudo de caso. Frederico WestphalenRS: UFSM, 2014. Disponível em: <http://w3.ufsm.br/frederico/images/Implementa\%C3\%A7\%C3\%A3o_do_MPS.BR_n a_empresa_Digifred_Sistemas_para_Gest\%C3\%A3o_P\%C3\%BAblica_Um_Estudo_d e_Caso.pdf>. Acesso em: jun. 2016.

CERATTI, S.; BERTOLINI, C. Governança de Tecnologia da Informação: um estudo na Universidade Federal de Santa Maria. Frederico Westphalen: UFSM, 2015. Disponível em:

$<$ http://w3.ufsm.br/frederico/images/GovernancadeTIumEstudonaUFSMCampusFrede ricoWestphalen.pdf $>$. Acesso em: ago. 2016.

CONCEIÇÃO, J.; SILVEIRA, S. R. Aplicação de Metodologias Ágeis para Desenvolvimento de Software: um estudo de caso na empresa Alliance Software. Frederico Westphalen-RS: UFSM, 2015. Disponível em: <http://w3.ufsm.br/frederico/images/AplicacaodeMetodologiasAgeisparaDesenvolvim entodeSoftwareumEstudodeCasonaEmpresaAllianceSoftware.pdf $>$. Acesso em: jun. 2016.

CNPq Conselho Nacional de Desenvolvimento Cientítico e Tecnológico. Grupo de Pesquisa IATE-UFSM. Disponível em: <dgp.cnpq.br/dgp/espelhogrupo/1534699540859088>. Acesso em agosto, 2016a.

CNPq - Conselho Nacional de Desenvolvimento Cientítico e Tecnológico. Grupo de Pesquisa Informática aplicada à Saúde. Disponível em: <dgp.cnpq.br/dgp/espelhogrupo/0162427575622397>. Acesso em: ago. 2016b.

CUNHA, M. I.; MARSICO, H. L; BORGES, F.A.; TAVARES, P. Inovações pedagógicas na formação inicial de professores. In: FERNANDES, C. M. B.; GRILLO, M. (Orgs.). Educação superior: travessias e atravessamentos. Canoas: Editora da ULBRA, 2001, p. 33-90.

FERRARI, R. A.; SILVEIRA, S. R; FREITAS, E. P. Ferramenta para Apoio ao Suporte Técnico em uma Rede FTTx empregando Raciocínio Baseado em Casos. Anais do VI EATI - Encontro Anual de Tecnologia da Informação, 2015. Disponível em: <http://eati.info/eati/2015/assets/anais/index.html>. Acesso em: mai. 2016.

FRIZON, M.; SILVEIRA, S. R.; CUNHA, G. B. Desenvolvimento de um Protótipo de Sistema Inteligente de Apoio à Decisão como Ferramenta de Business Intelligence: um estudo de caso na Arbaza Alimentos. RESIGET - Revista Eletrônica de Sistemas de Informação e de Gestão Tecnológica, v. 6, 2015. Disponível em: <http://periodicos.unifacef.com.br/index.php/resiget/article/view/1056>. Acesso em: jun. 2016. 
GARBIN, M.; SILVEIRA, S. R. Sistema de Recomendação Mobile: um estudo de caso para delivery. Anais do V EATI - Encontro Anual de Tecnologia da Informação, 2014. Disponível em: <http://eati.info/eati/2014/anais/>. Acesso em: mai. 2016.

GOERGEN, P. Universidade e Compromisso Social. In: RISTOFF, D.; SEVEGNANI, P. (Orgs.). Universidade e Compromisso Social. Brasília: INEP, 2006.

KLISZCZ, S.; SILVEIRA, S. R.; PARREIRA, F. J. Jogo Educacional Digital para apoio ao Ensino da Matemática. Revista TEAR, v. 5, n. 1, 2016.

LINKE, L. L.; SILVEIRA, S. R. Implantação de um Sistema de Help-Desk: um estudo de caso na Exatus Soluções Estratégicas. Frederico Westphalen-RS: UFSM, 2015. Disponível em: <http://w3.ufsm.br/frederico/images/ImplantacaodeumSistemadeHelpDeskumestudod ecasonaExatusSolucoesEstrategicas.pdf>. Acesso em: jun. 2016.

MEC - Ministério da Educação. Parecer CNE/CES 136/2012: diretrizes curriculares nacionais para os cursos de graduação em computação. 2012. Disponível em: $<$ http://portal.mec.gov.br/index.php?option=com_docman\&view=download\&alias $=11$ 205-pces136-11-pdf\&Itemid=30192>. Acesso em: jun. 2016.

MOERSCHBÄCHER, J. W.; SILVEIRA, S. R.; SANTOS, F. B. Criação de Lojas Virtuais por meio de Frameworks: um estudo de caso. Frederico Westphalen-RS: UFSM, 2015. $<\mathrm{http}: / /$ w3.ufsm.br/frederico/images/CriacaodeLojasVirtuaispormeiodeFramrworks.pd f>. Acesso em: jun. 2016.

MOLINA, D.; SILVEIRA, S. R.; SANTOS, F. B. Implantação de um Ambiente de Segurança de Redes de Computadores: um estudo de caso na Prefeitura Municipal de Palmeira das Missões-RS. Frederico Westphalen-RS: UFSM, 2015. Disponível em: $<$ http://w3.ufsm.br/frederico/images/ImplantacaodeumAmbientedeSegurancadeRedes deComputadoresUmEstudodeCasonaPrefeituradePalmeiradasMissoes.pdf $>$. Acesso em: jun. 2016.

MURARI, M. A.; CUNHA, G. B. Desenvolvimento de um Software para Migração de um Banco de Dados Relacional Firebird para o não relacional MongoDB. 2014. Disponível em: <http://w3.ufsm.br/frederico/images/Desenvolvimentodeumsoftwareparamigra\%C3\% A7\%C3\%A3odeumBancodeDadosRelacionalFirebirdparaon\%C3\%A3orelacionalMongoDB.pdf>. Acesso em: jun. 2016.

PARREIRA, F.; SILVEIRA, S. R.; BASSO, M.; KLISZCZ, S.; SOUZA, A. S. IATE: Inteligência Artificial e Tecnologia Educacional. Anais do VI EATI - Encontro Anual de Tecnologia da Informação, 2015. Disponível em: <http://eati.info/eati/2015/assets/anais/index.html>. Acesso em: mai. 2016.

PIMENTA, S. G.; ANASTASIOU, L. G. C. Docência no Ensino Superior. 3. ed. São Paulo: Cortez, 2008.

PIOVESAn, C. L.; BERTOLINI, C.; CUNHA, G. B. Processo de Classificação de Regras de Acessibilidade Web. Frederico Westphalen: UFSM, 2015. Disponível em: $<$ http://w3.ufsm.br/frederico/images/ProcessodeClassificacaodeRegrasdeAcessibilidad eWeb.pdf>. Acesso em: ago. 2016. 
PRADO; K. S.; SILVEIRA, S. R. Framework Genérico de Recomendação para Lojas Virtuais. RCT - Revista de Ciência e Tecnologia, v. 1, n. 1, 2015.

PREDIGER, D.; SILVEIRA, S. R.; FREITAS, E. P. Modelo de Aplicabilidade de Sistema RFID para Rastreabilidade na Indústria Alimentícia. RSC - Revista de Sistemas e Computação, v. 6, n. 1 , 2016. Disponível em: <http://www.revistas.unifacs.br/index.php/rsc/article/view/3620>. Acesso em: jul. 2016.

PEREIRA, L. A. C. A Rede Federal de Escolas Profissionalizantes. In: Universidade e Mundo do Trabalho. Organizado por: MOLL, J.; SEVEGNANI, P. Brasília: INEP, 2006.

PRETTZ, J. B.; BERTOLINI, C. Uma Proposta de um Processo de Teste em uma Empresa de Pequeno Porte. Revista JR. ICCEEg, n. 10. Disponível em: $<$ http://www.icceeg.c3.furg.br/index.php?Itemid=837\&option=bloco_texto\&id_site_c omponente=1241>. Acesso em: jul. 2016.

RIBEIRO, V. G.; ZABADAL, J. R. S. Pesquisa em Computação: uma abordagem metodológica para trabalhos de conclusão de curso e projetos de iniciação científica. Porto Alegre: UniRitter, 2010.

UFSM - Universidade Federal de Santa Maria. Projeto Pedagógico do Curso de Sistemas de Informação - Campus Frederico Westphalen-RS. 2016. Disponível em: <http://w3.ufsm.br/frederico/index.php/curso/graduacao/2-uncategorised/276-ppcsistemas-de-informacao >. Acesso em: jun. 2016.

UFSM - Universidade Federal de Santa Maria. Normatização do Trabalho de Graduação em Sistemas de Informação - Campus Frederico Westphalen-RS. 2013.

UFSM - Universidade Federal de Santa Maria. Projeto Político-Pedagógico. 2000.

UFSM - Universidade Federal de Santa Maria. Projeto Pedagógico do Curso de Sistemas de Informação - Campus Frederico Westphalen-RS. 2016. Disponível em: <http://w3.ufsm.br/frederico/index.php/curso/graduacao/2-uncategorised/276-ppcsistemas-de-informacao $>$. Acesso em: jun. 2016.

VIEIRA, J. P.; PARREIRA, F. J.; SILVEIRA, S. R. Desenvolvimento de um Sistema para Auxílio ao Diagnóstico de Diabetes empregando Redes Neurais Artificiais (SADD). RSC - Revista de Sistemas e Computação, v. 6, n. 1, 2016. Disponível em: <http://www.revistas.unifacs.br/index.php/rsc/article/view/4148>. Acesso em: jul. 2016.

ZACARIAS, I.; BERTOLINI, C.; FREITAS, E. P. Formal Analysis of a Bio-Inspired Wireless Sensor Network Routing Algorithm. Frederico Westphalen-RS: UFSM, 2015. Disponível em: $<$ http://w3.ufsm.br/frederico/images/FormalAnalysisofaBioInspiredWirelessSensorNet workRoutingAlgorithm.pdf>. Acesso em: jun. 2016.

ZANARDI, F., SILVEIRA, S. R. Um Estudo de Caso sobre o Perfil dos Profissionais e das Empresas de Tecnologia da Informação (TI) da Cidade de Frederico Westphalen - RS. Anais do Computer on the Beach, 2015. Disponível em: $<\mathrm{http}$ //siaiap32.univali.br/seer/index.php/acotb/article/view/7105>. Acesso em: jun. 2016. 